\title{
Genetic mapping of 15 human X chromosomal forensic short tandem repeat (STR) loci by means of multi-core parallelization
}

Toni Marie Diegoli ${ }^{1,2}$, Heinrich Rohde ${ }^{3}$, Stefan Borowski ${ }^{4}$, Michael Krawczak ${ }^{5}$, Michael D. Coble $^{6}$, Michael Nothnagel ${ }^{3, *}$

1 Office of the Chief Scientist, Defense Forensic Science Center, Ft. Gillem, GA, USA

2 Analytical Services, Inc., Arlington, VA, USA

3 Cologne Center for Genomics, University of Cologne, Cologne, Germany

4 Regional Computing Centre, University of Cologne, Cologne, Germany

5 Institute of Medical Informatics and Statistics, Christian-Albrechts University, Kiel, Germany

6 Applied Genetics Group, National Institute of Standards and Technology, Gaithersburg, MD, USA

* Correspondence address:

Michael Nothnagel

Cologne Center for Genomics, Department of Statistical Genetics and Bioinformatics

University of Cologne, Weyertal 115b, 50931 Cologne, Germany

Tel. : $\quad+49-221-478-96847$

E-mail: michael.nothnagel@uni-koeln.de

Keywords: X chromosome, genetic linkage, genetic mapping, short tandem repeat, forensic analysis, high performance computing 


\section{Abstract}

Typing of X chromosomal short tandem repeat (X STR) markers has become a standard element of human forensic genetic analysis. Joint consideration of many X STR markers at a time increases their discriminatory power but, owing to physical linkage, requires intermarker recombination rates to be accurately known. We estimated the recombination rates between 15 well established X STR markers using genotype data from 158 families (1041 individuals) and following a previously proposed likelihood-based approach that allows for single-step mutations. To meet the computational requirements of this family-based type of analysis, we modified a previous implementation so as to allow multi-core parallelization on a high-performance computing system. While we obtained recombination rate estimates larger than zero for all but one pair of adjacent markers within the four previously proposed linkage groups, none of the three X STR pairs defining the junctions of these groups yielded a recombination rate estimate of 0.50 . Corroborating previous studies, our results therefore argue against a simple model of independent $\mathrm{X}$ chromosomal linkage groups. Moreover, the refined recombination fraction estimates obtained in our study will facilitate the appropriate joint consideration of all 15 investigated markers in forensic analysis. 


\section{Introduction}

Typing of X chromosomal short tandem repeat (X STR) markers has become a standard element of human forensic genetic analysis. X STRs are characterized by a number of features that render them particularly useful for forensic purposes, namely their specific mode of inheritance and their ease of haplotype inference due to male hemizygosity. The latter is important because joint consideration of many X STRs at a time increases their discriminatory power, but at the same time requires inter-marker recombination rates to be known [1].

Various X STR marker sets have been developed for routine forensic use. This includes the Mentype ${ }^{\circledR}$ Argus X-8 PCR amplification kit [2, 3], featuring markers DXS10135, DXS8378, DXS7132, DXS10074, HPRTB, DXS10101, DXS10134 and DXS7423, and its extension to the Investigator ${ }^{\circledR}$ Argus X-12 PCR amplification kit (Qiagen, Hilden, Germany), which additionally includes DXS10148, DXS10079, DXS10103 and DXS10146 [4-8]. Noncommercial X STR marker sets have been developed by individual laboratories [9-21]. One of these comprises 15 X STR markers [22] including the four markers (DXS8378, DXS7132, HPRTB and DXS7423) that were present in the only commercially available kit at the time, namely the Mentype ${ }^{\circledR}$ Argus X-UL kit [3], and that have been retained in subsequent versions of the kit, i.e. in Mentype ${ }^{\circledR}$ Argus X-8 and Investigator ${ }^{\circledR}$ X-12. The 15 -marker set in question includes 11 additional markers (DXS9902, DXS6795, DXS6803, DXS6789, DXS7424, DXS101, GATA172D05, DXS7130, GATA165B12, GATA31E08 and DXS10147). It is amplified in two short-amplicon multiplexes for use with degraded DNA. Similar to the commercial kits, the 15-marker customized set systematically includes STRs located in one of the four linkage groups (LGs) presumed at the time of design to make up the $\mathrm{X}$ chromosome [23]. Unlike the commercial kits, however, the markers chosen for this set are located at 
greater physical distances from each other, thereby implying a greater possibility of intragroup recombination.

Genetic markers on the $\mathrm{X}$ chromosome are not stochastically independent, in principle, owing to their physical linkage. For practical purposes, X STRs were therefore assigned to LGs (Table 1) that were assumed to be unlinked whilst recombination within groups was deemed negligible [23, 24]. However, subsequent analysis of comprehensive family data challenged this view [25, 26] and inspired a recent study [27] that was aimed at estimating the intermarker recombination rates for the Investigator ${ }^{\circledR}$ Argus X-12 kit, using a likelihood-based approach that allows for single-step mutations. Indeed, whilst LGs I and II were found to be unlinked, groups II, III and IV showed substantial inter-group linkage. Moreover, markers located within all four LGs showed non-negligible recombination, i.e. they were not completely linked. These results partially agreed with previous studies and complied with the Rutgers genetic map [26, 28-30].

Here, we set out to estimate the inter-marker recombination rates for the above mentioned set of 15 X STRs, using the likelihood-based approach reported before [27]. However, the large number of markers included in the present study rendered the computational complexity of the task prohibitive for the original implementation. We therefore modified the approach by code parallelization to allow all necessary calculations to be performed on a high-performance computing cluster. In this way, parallelization proved itself both a requirement and a facilitator of the full exploitation of $\mathrm{X}$ chromosomal multi-marker genotypes in forensic practice. 


\section{Materials and Methods}

\section{Family samples}

Two types of families were recruited from different sources (see below) and classified following a previously published approach [27]. Type I families were three-generation families comprising a maternal grandfather, one or more mothers and their son(s). If more than one mother was present in a family, the family was split into sub-families comprising the maternal grandfather, a single mother and all of her sons. Type II families comprised a single mother and two or more sons. We also considered families where information on the father and at least one daughter was available, and where the X chromosomal haplotypes of the daughter(s) could be inferred unequivocally. Such families were transformed into type I and type II format, respectively, by masking the paternally inherited X chromosome of the daughter(s), thereby effectively turning them into 'sons', and subsequently discarding the paternal genetic information. Complex pedigrees comprising several generations and multiple offspring could thus comprise sub-families of different type.

Family data appropriate for recombination rate estimation were obtained from three different sources. First, commercially available DNA extracts from 61 extended families included in the Centre d'Étude du Polymorphisme Humain (CEPH) panel were purchased from the NIGMS Human Genetic Cell Repository at the Coriell Institute for Medical Research (https://catalog.coriell.org/1/NIGMS), comprising a total of 545 individuals from 52 families (43 type I and 9 type II). Second, we considered genotypes previously generated for a mutation rate study [31] of non-excluded paternity cases that involved more than one child, adding 72 type II families comprising 312 individuals to our study. Finally, we drew upon 126 individuals belonging to one of 34 different sub-families (11 type I and 23 type II) from six 
extended U.S. families of European ancestry previously typed at autosomal STR markers [32]. In total, 158 families (54 type I and 104 type II) were included in our study. The project was reviewed and approved by the U.S. Army Medical Research and Materiel Command Institutional Review Board Office.

\section{DNA genotyping}

All individuals were genotyped for 15 X STR markers, namely DXS6795, DXS9902, DXS8378, DXS7132, DXS6803, DXS6789, DXS7424, DXS101, GATA172D05, DXS7130, GATA165B12, HPRTB, GATA31E08, DXS10147 and DXS7423, using two mini X STR multiplexes according to a previously described protocol [22].

\section{Quality control}

Since the conversion of daughter profiles into mock 'son profiles' required manual manipulation of the data, additional quality control measures were implemented so as to ensure that the resulting dataset was error-free. First, all meioses were screened for possible mutations using a spreadsheet program. In particular, for daughters-turned-sons and their mothers, a mutation could indicate either a mistake in the process of removing the paternal contribution or a true maternal mutation. Errors were corrected by referring to the original unedited profiles, and true mutations were categorized as either maternal, paternal, or of unknown origin. Mutations of unknown origin discovered in daughters-turned-sons were considered paternal in order to allow their inclusion in the linkage analysis. Since the linkage analysis scripts did not tolerate mutations of more than one step, or mutations between maternal grandfathers and mothers, the respective markers were rendered uninformative for linkage in these rare cases. Other mutations that would not impact upon the linkage analysis or that could be determined to be maternal were maintained in the dataset. Table 2 
summarizes the genotypic information finally available for the estimation of inter-marker recombination rates.

\section{Estimation of inter-marker recombination rates}

We followed a recently published likelihood approach to simultaneously estimate intermarker recombination rates for multiple STR markers, allowing for possible single-step mutations in the process [27]. Briefly, the overall log-likelihood was set equal to the sum of family-wise likelihoods. While the grandpaternal genotype information allowed phasing of maternal genotypes in type I families and, consequently, accelerated calculation of the family likelihood, phase information was not available in type II families so that all possible haplotype constellations had to be considered in these families. To this end, we assigned equal prior probabilities to all haplotype constellations, thereby ignoring possible linkage disequilibrium within LGs as before [27]. Likelihood maximization was performed using statistics software R v3.1.1 [33] via in-house scripts incorporating the optim function with the L-BFGS-B method [34] to allow for box constrains on the recombination parameters. Recombination rates were allowed to vary between $10^{-8}$ and 0.5 in order to avoid numerical underflow. The one-sided mutation rate was set equal to $10^{-3}$ for all markers [27]. We also investigated the effect of increasing this value to $2.5 \times 10^{-3}$, which yielded virtually identical results (data not shown).

Following the same strategy as in the original report of our approach [27], three different sets of starting values for the inter-marker recombination rates ('schemes') were chosen for each maximization round.

1. Interpolated: Recombination rates were interpolated from known physical intermarker distances (Table 1). Genetic distances were approximated by a commonly used rule-of-thumb, whereby $1 \mathrm{Mb}$ of DNA roughly corresponds to $1 \mathrm{cM}$, and then 
converted to recombination rates using the Kosambi mapping function [35]. This scheme incorporates the maximum amount of external information available while ignoring the concept of LGs altogether.

2. Flat: All recombination rates between adjacent markers were set equal to 0.25 . In this way, no prior assumptions about relative map positions were made at all.

3. Blocks: Complete linkage $\left(\theta=10^{-8}\right)$ was assumed within LGs and free recombination $(\theta=0.5)$ was assumed to occur between LGs. This scheme emulates the originally conceived situation of four independent super-informative markers.

The uncertainty of the recombination rate estimates was quantified by $95 \%$ support intervals [27]. More specifically, a support interval includes all $\theta$ values that would not be deemed significantly different from the MLE in a two-sided one degree-of-freedom likelihood-ratio test at the 0.05 significance level, except for MLEs equal to 0.0 or 0.5 (i.e., the limiting values of $\theta$ ) where a one-sided test was used.

\section{Multi-core parallelization}

Type II families, where iteration over all possible haplotype constellations in the mother was required, turned out to be intractable by sequential likelihood calculation. Therefore, we decided to explore alternative means of implementing our linkage analysis approach.

Likelihood maximization with $\mathrm{R}$ function optim draws upon iterative calculations of the likelihood function. This function is compiled from a $\mathrm{C}$ kernel consisting mainly of a multiple loop nest with numerous iterations that process a small amount of data over and over again. The function is made available to $\mathrm{R}$ by loading the respective shared object. Since the number of possible haplotype constellation increases exponentially with the number of markers, the calculation of the likelihood function takes an enormous amount of computing time on a comparatively small memory footprint. However, each maternal haplotype constellation and, 
therefore, all iterations of the multiple loop nest make independent contributions to the family likelihood.

We therefore resorted to parallelization of the original code [27] for it to be run on a highperformance computing (HPC) cluster (scripts are available from the authors upon request). More specifically, we introduced OpenMP (http://www.openmp.org) parallelization in an outer loop level to utilize Symmetric Multiprocessing (SMP) compute nodes with multiple processors. Since the outermost loop, i.e. the one running over pedigrees, did not involve enough iterations to provide sufficient load balancing between executing OpenMP threads in dynamic work sharing, we moved the work-sharing to the next loop, i.e. the one running over haplotype constellation. This loop afforded many more iterations than needed for perfect load balancing, even on big SMP nodes with many cores. Since the loop is still an outer loop, parallel overhead remained limited. Finally, summation of thread-wise contributions was realized as a reduction operation outside the work-sharing loop.

In production, our implementation achieved considerable speedup for 32 threads running on an SMP node with 32 cores. However, some computational tasks still could not be finished within a single batch job. To overcome this problem, likelihood maximization had to be restarted upon saving and loading the current workspace in the R script. We validated our efforts by comparing the results of certain computational steps of the original single-core and the parallelized multi-core implementation. Using subsets of the full data set, the two implementations yielded virtually identical results (data not shown). We also applied the parallelized software to two previously analyzed data sets comprising 8 and 12 markers [27], respectively. Again, the results were virtually identical to the original recombination fraction estimates, with only a few marker pairs showing minor deviations of the order of $10^{-3}$ to $10^{-4}$. Graphs were created using R v3.2.2 [36]. 


\section{Results}

The three different sets (schemes) of starting values for the recombination rate estimation led to virtually identical results. Within linkage groups (LGs), $\theta$ estimates ranged from zero (marker pairs DXS7424-DXS101 and DXS10147-DXS7423) to 0.2186 (marker pair GATA31E08-DXS10147). Between adjacent LGs, estimates varied between 0.1117 (LGs III and IV) and 0.4521 (LGs I and II) (Table 3). In fact, none of the three marker pairs at the junction of two neighboring LGs yielded a recombination rate estimate of 0.50 , which would have indicated complete lack of linkage.

The recombination rate estimates were well correlated with the physical distance between markers except for three notable violations of the original LG model (Figure 1). First, although located within one and the same LG, markers GATA31E08 and DXS10147 yielded a recombination rate estimate of 0.2186 , which was much higher than other rates estimated at similar physical distance (i.e., $9.429 \mathrm{Mb}$ ). Moreover, the corresponding 95\% support interval (0.1771-0.2648) does not include rates that would appear compatible with close linkage. Further studies are therefore necessary to clarify whether GATA31E08-DXS10147 indeed represents a recombination hot spot. Second, we noted strongly reduced recombination between LGs II and III, and LGs III and IV, compared to free recombination.

Our results agree well with those of four previous studies $[26,30,37,38]$ despite the fact that, in those studies, other markers defined the LG junctions than in ours (Table 3). As regards the possibility of recombination hotspots, for example, the recombination rate between DXS10101 (physical location at $133.654 \mathrm{Mb}$; genetic location at $150.02 \mathrm{cM}$ ) and DXS10134 (149.650 Mb; $184.34 \mathrm{cM}$ ) was reported before to equal 0.25 (95\% support interval: $0.17-0.35$ ) which is almost twice the theoretically expected value of 0.136 [26]. An increased 
recombination rate of 0.434-0.367 was also reported for markers DXS10101 and DXS10146 $(149.335 \mathrm{Mb} ; 184.10 \mathrm{cM})$ [30, 37]. Taken together, the available data thus suggest locally increased recombination at Xq27 in a region that may well extend to include distal markers GATA31E08 and DXS10147.

\section{Discussion}

Only few studies have been performed to date to systematically assess the recombination rates between X STRs, and none of these studies has been as comprehensive as the present one. In any case, most previous efforts involved marker sets that overlapped widely with the $15 \mathrm{X}$ STRs studied here, including an analysis of 32 two-generation families using the Argus X-8 kit [26]. However, the mothers included in that study could not have their X chromosomal haplotypes confirmed because no grandparents were included. Therefore, two possible haplotypes had to be allowed for in each child, and this ambiguity had to be accounted for in the linkage analysis. Interestingly, a recombination rate of 0.45 was inferred in that study between linkage groups (LGs) I and II that agrees very well with our own result $(0.4521)$ despite the fact that the border of LG I was defined by different markers (DXS8378 and DXS6795).

Evidence for free recombination between LGs I and II had been provided by another study of $20 \mathrm{X}$ STRs in 80 two-generation families [39], where a recombination rate of 0.49980 was estimated for junction markers DXS8378 and DXS7132. Also in agreement with our findings, free recombination was found to be lacking between LGs II and III and between LGs III and IV [39], despite different junction markers being used compared to our study. Thus, while a 
model of independent LGs on the X chromosome may be appropriate for LGs I and II, it is definitely not valid in the case of the other, previously proposed LGs. Moreover, the introduction of new markers between LGs I and II will surely render the concept of freely recombining marker groups invalid in this chromosomal region as well.

The analytical approach taken here was first described in connection with a linkage study of 12 markers in two- and three-generation families [27]. The results of that study agree widely with the present ones in terms of the inter-group recombination rates between LGs I and II (almost free recombination) and between LGs III and IV (reduced recombination). However, even though the older study yielded a recombination rate $<0.5$ between LGs II and III (0.4252) as well, the observed lack of free recombination was not as dramatic as in the present study, where the recombination rate estimate equaled 0.1135 . In our view, this discrepancy is most likely due to different junction markers being used to define the two LGs (DXS10101DXS10146 in [27] vs. GATA165B12-HPRTB here). Moreover, none of the internal marker pairs of the respective LGs overlapped between the two studies, thereby hampering any direct comparison between the two studies even further.

For one marker pair examined in the present study, discordant results (i.e., recombination rate estimates falling outside our $95 \%$ support intervals) have been reported before. Thus, a study of 39 X STRs in 90 three-generation pedigrees with a goal to assess linkage between four groups of physically close markers [37] revealed a significantly higher recombination rate for DXS7424-DXS101 than estimated here (Table 3). Similarly, a recent study of 25 marker pairs in two- and three-generation pedigrees investigated DXS7424-DXS101 [38], and the respective $95 \%$ support interval for the recombination rate estimate did not overlap with that obtained in our study. This discrepancy is explicable in terms of the negligence of single-step mutation in all but the present study, although this does not exclude other explanations. More 
work is necessary to clarify this issue, including the re-analysis of previously published data following our mutation-sensitive approach.

The computational burden of estimating inter-marker recombination rates with our likelihoodbased algorithm increased dramatically by the mere consideration of three additional markers, and eventually rendered it prohibitive for running on a single compute core. We solved this problem by Symmetric Multiprocessing (SMP) on 32 cores of a single high-performance computing (HPC) node. Nevertheless, runtime still amounted to several months. Therefore, our approach is probably not suitable for the investigation of substantially larger marker sets, at least not at the current speed of HPC nodes. Multiple nodes with some form of information exchange between the nodes would be required instead, such as master-slave settings with a message-passing interface (MPI; http://www.mpi-forum.org).

Throughout our study, we have assumed that the STRs under consideration lack allelic correlation, or linkage disequilibrium (LD), which is a standard assumption underlying many statistical genetics approaches. However, while limited LD between X STR markers may exist, its incorporation into a particular statistical model would not be straightforward. Moreover, consideration of LD may actually impair the accuracy and precision of recombination rate estimates owing to possible population differences in LD structure. In any case, we surmise that ignorance of LD has had only minor impact on the results presented here because of the high mutation rate of STRs that would rapidly reduce or even eradicate any existing $\mathrm{LD}$.

We previously emphasized the importance of linkage for the interpretation of X STR data and the consequent need for large collaborative studies to determine relevant recombination rates empirically [27]. The present study has added to meeting this demand by a large-scale analysis of multi-generation pedigrees, including $61 \mathrm{CEPH}$ panel families that are freely 
available to the scientific community from the Coriell repository. Given the importance of linkage for the proper interpretation of multi-marker genotype data in forensic casework, we surmise that the computational strategy described here will become relevant for future forensic applications of next generation DNA sequencing as well. 


\section{Competing interests}

The authors declare that they have no competing interests.

\section{Acknowledgements}

The authors thank Minh Nguyen, Chad Ernst, and the National Institute of Justice for grant support and management; Dr. Peter Vallone, Dr. John Butler, Erica Butts, Dr. Moses Schanfield, and Patty Czarnecki for assistance with sample extracts and the provision of associated quantification data; Jessica Saunier for bioinformatics assistance; and Col. Louis Finelli, James Canik, Dr. Timothy McMahon, Cynthia Thomas, the Armed Forces DNA Identification Laboratory, and the American Registry of Pathology for logistical and administrative support. We also thank the Regional Computing Center of the University of Cologne (RRZK) for computing time on the DFG-funded High Performance Computing (HPC) system CHEOPS. This project was supported in part by Award No. 2011-DN-BXK401 by the National Institute of Justice, Office of Justice Programs, U.S. Department of Justice to TMD and MDC. Certain commercial equipment, instruments, or materials (or suppliers, or software) are identified in this paper to foster understanding. Such identification does not imply recommendation or endorsement by the National Institute of Standards and Technology, nor does it imply that the materials or equipment identified are necessarily the best available for the purpose. The opinions, findings, and conclusions or recommendations expressed in this publication are those of the authors and are not to be construed as official or as reflecting those of the Department of Justice, Department of Commerce, Department of the Army, or Department of Defense. None of the funding organizations had any influence on the design, conduct or conclusions of the study. The authors declare no competing financial interests. 


\section{References}

[1] M Krawczak. Kinship testing with X-chromosomal markers: mathematical and statistical issues. Forensic Sci Int Genet. 2007;1:111-4.

[2] D Becker, H Rodig, C Augustin, J Edelmann, F Gotz, S Hering, et al. Population genetic evaluation of eight X-chromosomal short tandem repeat loci using Mentype Argus X-8 PCR amplification kit. Forensic science international Genetics. 2008;2:69-74.

[3] BiotypeAG. Mentype ${ }^{\circledR}$ Argus X-8 PCR Amplification Kit Manual. 2007.

[4] J Edelmann, S Hering, C Augustin, R Szibor. Characterisation of the STR markers DXS10146, DXS10134 and DXS10147 located within a $79.1 \mathrm{~kb}$ region at Xq28. Forensic Sci Int Genet. 2008;2:41-6.

[5] S Hering, C Augustin, J Edelmann, M Heidel, J Dressler, H Rodig, et al. DXS10079, DXS10074 and DXS10075 are STRs located within a 280-kb region of Xq12 and provide stable haplotypes useful for complex kinship cases. International journal of legal medicine. 2006;120:337-45.

[6] T Hundertmark, S Hering, J Edelmann, C Augustin, I Plate, R Szibor. The STR cluster DXS10148-DXS8378-DXS10135 provides a powerful tool for X-chromosomal haplotyping at Xp22. Int J Legal Med. 2008;122:489-92.

[7] H Rodig, F Kloep, L Weissbach, C Augustin, J Edelmann, S Hering, et al. Evaluation of seven X-chromosomal short tandem repeat loci located within the Xq26 region. Forensic science international Genetics. 2010;4:194-9.

[8] Qiagen. Investigator Argus X-12 Handbook. 2010.

[9] H Asamura, H Sakai, K Kobayashi, M Ota, H Fukushima. MiniX-STR multiplex system population study in Japan and application to degraded DNA analysis. Int J Legal Med. 2006;120:174-81.

[10] H Asamura, H Sakai, M Ota, H Fukushima. Japanese population data for eight X-STR loci using two new quadruplex systems. Int J Legal Med. 2006;120:303-9.

[11] D Athanasiadou, B Stradmann-Bellinghausen, C Rittner, PM Schneider. Development of a quadruplex PCR system for the genetic analysis of X-chromosomal STR loci. International Congress Series 1239. 2003;311-314.

[12] I Gomes, M Prinz, R Pereira, C Meyers, RS Mikulasovich, A Amorim, et al. Genetic analysis of three US population groups using an X-chromosomal STR decaplex. Int J Legal Med. 2007;121:198-203.

[13] L Gusmao, P Sanchez-Diz, C Alves, I Gomes, MT Zarrabeitia, M Abovich, et al. A GEPISFG collaborative study on the optimization of an X-STR decaplex: data on 15 Iberian and Latin American populations. Int J Legal Med. 2009;123:227-34.

[14] L Henke, M Dulmer, J Henke. A pentaplex PCR assay for the genetic analysis of ChrX short tandem repeat (STR) loci. International Congress Series 1261. 2004;266-268.

[15] C Bini, S Ceccardi, G Ferri, S Pelotti, M Alu, E Roncaglia, et al. Development of a heptaplex PCR system to analyse X chromosome STR loci from five Italian population samples. A collaborative study. International Congress Series 1261. 2004;272-274. 
[16] M Poetsch, H Petersmann, A Repenning, E Lignitz. Development of two pentaplex systems with X-chromosomal STR loci and their allele frequencies in a northeast German population. Forensic Sci Int. 2005;155:71-6.

[17] EM Ribeiro Rodrigues, FP Leite, MH Hutz, J Palha Tde, AK Ribeiro dos Santos, SE dos Santos. A multiplex PCR for $11 \mathrm{X}$ chromosome STR markers and population data from a Brazilian Amazon Region. Forensic Sci Int Genet. 2008;2:154-8.

[18] C Robino, A Giolitti, S Gino, C Torre. Development of two multiplex PCR systems for the analysis of $12 \mathrm{X}$-chromosomal STR loci in a northwestern Italian population sample. Int J Legal Med. 2006;120:315-8.

[19] S Turrina, R Atzei, G Filippini, D De Leo. Development and forensic validation of a new multiplex PCR assay with $12 \mathrm{X}$-chromosomal short tandem repeats. Forensic Sci Int Genet. 2007;1:201-4.

[20] MT Zarrabeitia, T Amigo, C Sanudo, A Zarrabeitia, D Gonzalez-Lamuno, JA Riancho. A new pentaplex system to study short tandem repeat markers of forensic interest on $\mathrm{X}$ chromosome. Forensic Sci Int. 2002;129:85-9.

[21] KA Tabbada, MC De Ungria, LP Faustino, D Athanasiadou, B Stradmann-

Bellinghausen, PM Schneider. Development of a pentaplex X-chromosomal short tandem repeat typing system and population genetic studies. Forensic Sci Int. 2005;154:173-80.

[22] TM Diegoli, MD Coble. Development and characterization of two mini-X chromosomal short tandem repeat multiplexes. Forensic Sci Int Genet. 2011;5:415-21.

[23] R Szibor, M Krawczak, S Hering, J Edelmann, E Kuhlisch, D Krause. Use of X-linked markers for forensic purposes. Int J Legal Med. 2003;117:67-74.

[24] R Szibor. X-chromosomal markers: past, present and future. Forensic Sci Int Genet. 2007;1:93-9.

[25] FB Machado, E Medina-Acosta. Genetic map of human X-linked microsatellites used in forensic practice. Forensic Sci Int Genet. 2009;3:202-4.

[26] AO Tillmar, P Mostad, T Egeland, B Lindblom, G Holmlund, K Montelius. Analysis of linkage and linkage disequilibrium for eight X-STR markers. Forensic Sci Int Genet. 2008;3:37-41.

[27] M Nothnagel, R Szibor, O Vollrath, C Augustin, J Edelmann, M Geppert, et al. Collaborative genetic mapping of 12 forensic short tandem repeat (STR) loci on the human X chromosome. Forensic Sci Int Genet. 2012;6:778-84.

[28] TC Matise, F Chen, W Chen, FM De La Vega, M Hansen, C He, et al. A secondgeneration combined linkage physical map of the human genome. Genome Res. 2007; 17:1783-6.

[29] C Tomas, V Pereira, N Morling. Analysis of 12 X-STRs in Greenlanders, Danes and Somalis using Argus X-12. Int J Legal Med. 2012;126:121-8.

[30] H Pamjav, R Kugler, A Zalan, A Volgyi, Z Straky, P Endredy, et al. X chromosomal recombination study in three-generation families in Hungary. Forensic Sci Int Genet. 2012;6:e95-6.

[31] TM Diegoli, A Linacre, MS Schanfield, MD Coble. Mutation rates of 15 X chromosomal short tandem repeat markers. Int J Legal Med. 2014;128:579-87.

[32] KL O'Connor, AO Tillmar. Effect of linkage between vWA and D12S391 in kinship analysis. Forensic Sci Int Genet. 2012;6:840-4. 
[33] R Development Core Team. R: A language and environment for statistical computing. R Foundation for Statistical Computing, Vienna, Austria2014.

[34] RH Byrd, P Lu, J Nocedal, C Zhu. A limited memory algorithm for bound constrained optimization. SIAM J Scientific Computing. 1995;16:1190-208.

[35] DD Kosambi. The estimation of the map distance from recombination values. Ann Eugen. 1944;12:172-5.

[36] R Development Core Team. R: A language and environment for statistical computing. R Foundation for Statistical Computing, Vienna, Austria2015.

[37] S Hering, J Edelmann, C Augustin, E Kuhlisch, R Szibor. X chromosomal recombination--a family study analysing 39 STR markers in German three-generation pedigrees. Int J Legal Med. 2010;124:483-91.

[38] QL Liu, ZD Li, CT Li, H Zhao, YD Wu, Q Li, et al. X chromosomal recombination--a family study analyzing $26 \mathrm{X}-\mathrm{STR}$ Loci in Chinese Han three-generation pedigrees.

Electrophoresis. 2013;34:3016-22.

[39] S Inturri, S Menegon, A Amoroso, C Torre, C Robino. Linkage and linkage disequilibrium analysis of X-STRs in Italian families. Forensic Sci Int Genet. 2011;5:152-4.

[40] AQJ Nato, S Buyske, TC Matise. The Rutgers Map: A third-generation combined linkage-physical map of the human genome. Human Genetics of New Jersey Second Research Day. Life Sciences Building, Rutgers University, Piscataway, NJ, USA.2012. 


\section{Figures}

Figure 1: Pair-wise physical and genetic distances between adjacent X STR markers.

See Table 3 for point estimates of the recombination rates. Circles: intra-linkage group (LG)

marker pairs; diamonds: inter-LG marker pairs; dashed line: theoretically expected

recombination rate, derived from the $1 \mathrm{Mb}=1 \mathrm{cM}$ approximation and using the Kosambi mapping function [35]. Ideally, recombination rates should be close to zero for intra-LG pairs, and close to 0.5 for inter-LG pairs. At true recombination hotspots, the recombination rate may cross the dashed line. Marker pairs with unusual recombination rates are labeled (see text for further details). 
Table 1: Physical and genetic localisation of 15 X STRs

\begin{tabular}{|c|c|c|c|c|}
\hline Marker & $\begin{array}{c}\text { Linkage } \\
\text { group }\end{array}$ & $\begin{array}{l}\text { Cytogenetic } \\
\text { localisation }\end{array}$ & $\begin{array}{r}\text { Physical } \\
\text { localization }[\mathrm{Mb}]^{\mathrm{b}}\end{array}$ & $\begin{array}{l}\text { Genetic } \\
\text { localization }[\mathrm{cM}]\end{array}$ \\
\hline DXS8378 & I & $\mathrm{Xp} 22.31$ & 9.370 & $20.58^{c}$ \\
\hline DXS9902 & & $\mathrm{Xp} 22.2$ & 15.324 & $32.71^{\mathrm{c}}$ \\
\hline DXS6795 & & $\mathrm{Xp} 22.11$ & 23.245 & $44.51^{\mathrm{c}}$ \\
\hline DXS7132a & II & $\mathrm{Xq12}$ & 64.656 & $91.23^{c}$ \\
\hline DXS6803 & & $\mathrm{Xq} 21.31$ & 86.431 & $99.63^{c}$ \\
\hline DXS6789 & & $\mathrm{Xq} 21.33$ & 95.450 & $108.81^{\mathrm{c}}$ \\
\hline DXS7424 & & $\mathrm{Xq} 22.1$ & 100.619 & $115.45^{\mathrm{c}}$ \\
\hline DXS101 & & $\mathrm{Xq} 22.1$ & 101.413 & $116.40^{\mathrm{d}}$ \\
\hline GATA172D05 & & $\mathrm{Xq} 23$ & 113.175 & $124.67^{\mathrm{c}}$ \\
\hline DXS7130 & & $\mathrm{Xq} 24$ & 118.200 & $130.72^{c}$ \\
\hline GATA165B12 & & $\mathrm{Xq} 24$ & 120.888 & $136.51^{\mathrm{d}}$ \\
\hline HPRTB $^{\mathrm{a}}$ & III & $\mathrm{Xq} 26.2$ & 133.616 & $149.96^{\mathrm{d}}$ \\
\hline GATA31E08 & IV & $\mathrm{Xq} 27.2$ & 140.234 & $160.91^{\mathrm{d}}$ \\
\hline DXS10147 & & $\mathrm{Xq} 28$ & 149.663 & $184.39^{\mathrm{d}}$ \\
\hline DXS7423 & & $\mathrm{Xq} 28$ & 149.711 & $184.57^{\mathrm{c}}$ \\
\hline
\end{tabular}

Physical and genetic localizations are given according to NCBI build 37 or the Rutgers Map v3 [40], respectively. a: also included in the Investigator ${ }^{\circledR}$ Argus X-12 kit; b: localization according to BLAT in silico PCR search using the Feb. 2009 assembly; c: derived from recombination rate estimates provided by the Rutgers Map v3 (NCBI build 36), using the Kosambi mapping function [35]; d: linear interpolation from physical distances between adjacent markers in the Rutgers Map v3. 
Table 2: X STR genotype data used for linkage and mutation analysis

\begin{tabular}{|c|c|c|c|}
\hline \multirow[t]{2}{*}{ Marker $^{\mathrm{a}}$} & \multicolumn{2}{|c|}{$\begin{array}{l}\text { Number of linkage-informative } \\
\text { meioses }\end{array}$} & \multirow{2}{*}{$\begin{array}{c}\text { Number of possible } \\
\text { (unambiguous) } \\
\text { mutations }\end{array}$} \\
\hline & Type I families & Type II families & \\
\hline DXS8378 & 262 & 240 & $330(0)$ \\
\hline DXS9902 & 282 & 234 & $321(3)$ \\
\hline DXS6795 & 250 & 258 & $86(0)$ \\
\hline DXS7132 ${ }^{\mathrm{a}}$ & 247 & 238 & $290(0)$ \\
\hline DXS6803 & 318 & 271 & $194(0)$ \\
\hline DXS6789 & 236 & 255 & $268(0)$ \\
\hline DXS7424 & 261 & 284 & $265(0)$ \\
\hline DXS101 & 279 & 294 & $105(0)$ \\
\hline GATA172D05 & 271 & 264 & $161(0)$ \\
\hline DXS7130 & 221 & 257 & $180(0)$ \\
\hline GATA165B12 & 202 & 183 & $237(0)$ \\
\hline HPRTB $^{\mathrm{a}}$ & 266 & 246 & $315(0)$ \\
\hline GATA31E08 & 263 & 270 & $190(0)$ \\
\hline DXS10147 & 242 & 210 & $180(0)$ \\
\hline DXS7423 ${ }^{\mathrm{a}}$ & 226 & 232 & $251(0)$ \\
\hline
\end{tabular}

a: also included in the Investigator ${ }^{\circledR}$ Argus X-12 kit; b: total number of sons with a heterozygous mother. 
Table 3: Estimates of the recombination rate between adjacent X STRs

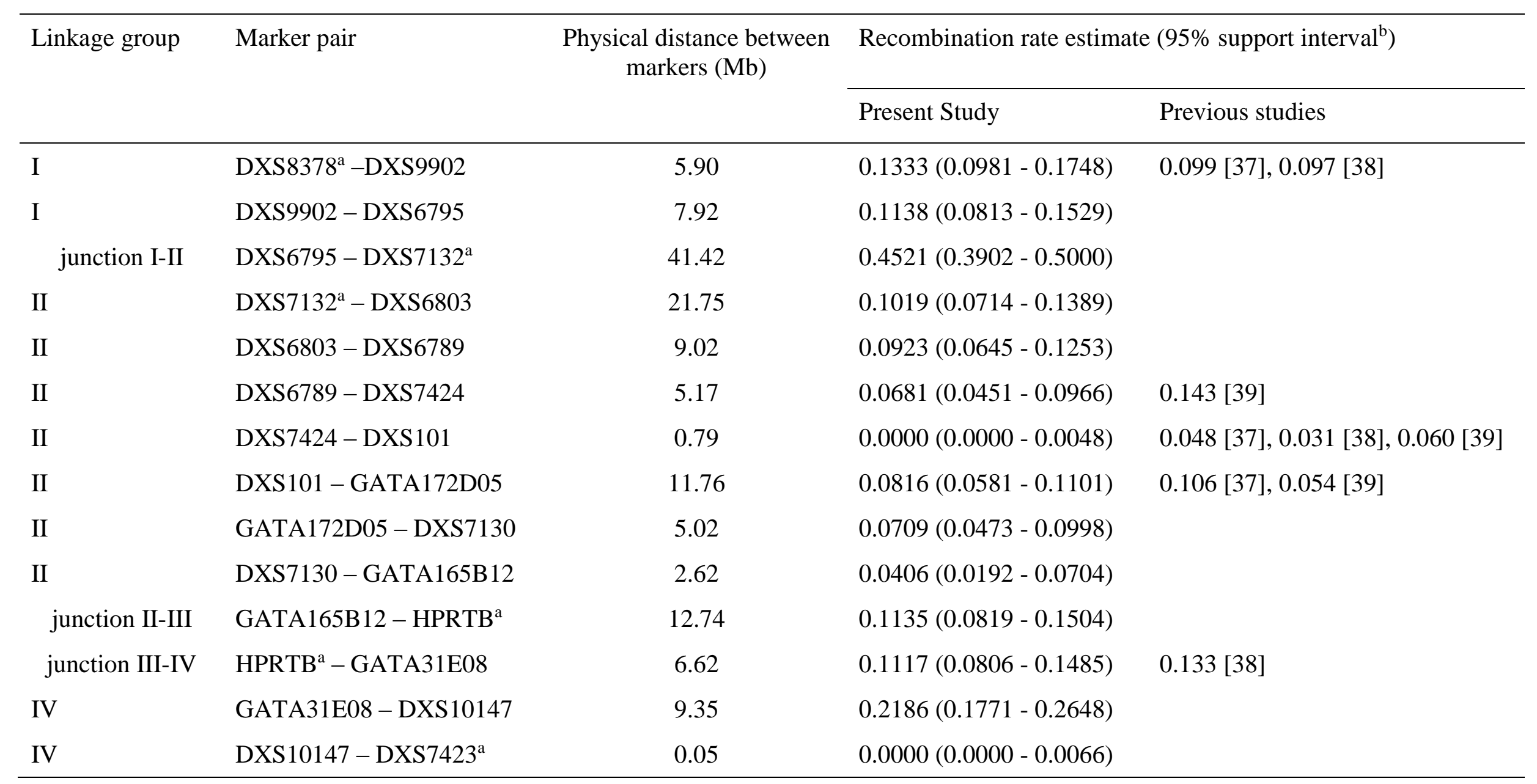

a: also included in the Investigator ${ }^{\circledR}$ Argus X-12 kit; b: one-sided 95\% support interval for marker pairs with a maximum likelihood estimate of zero. 


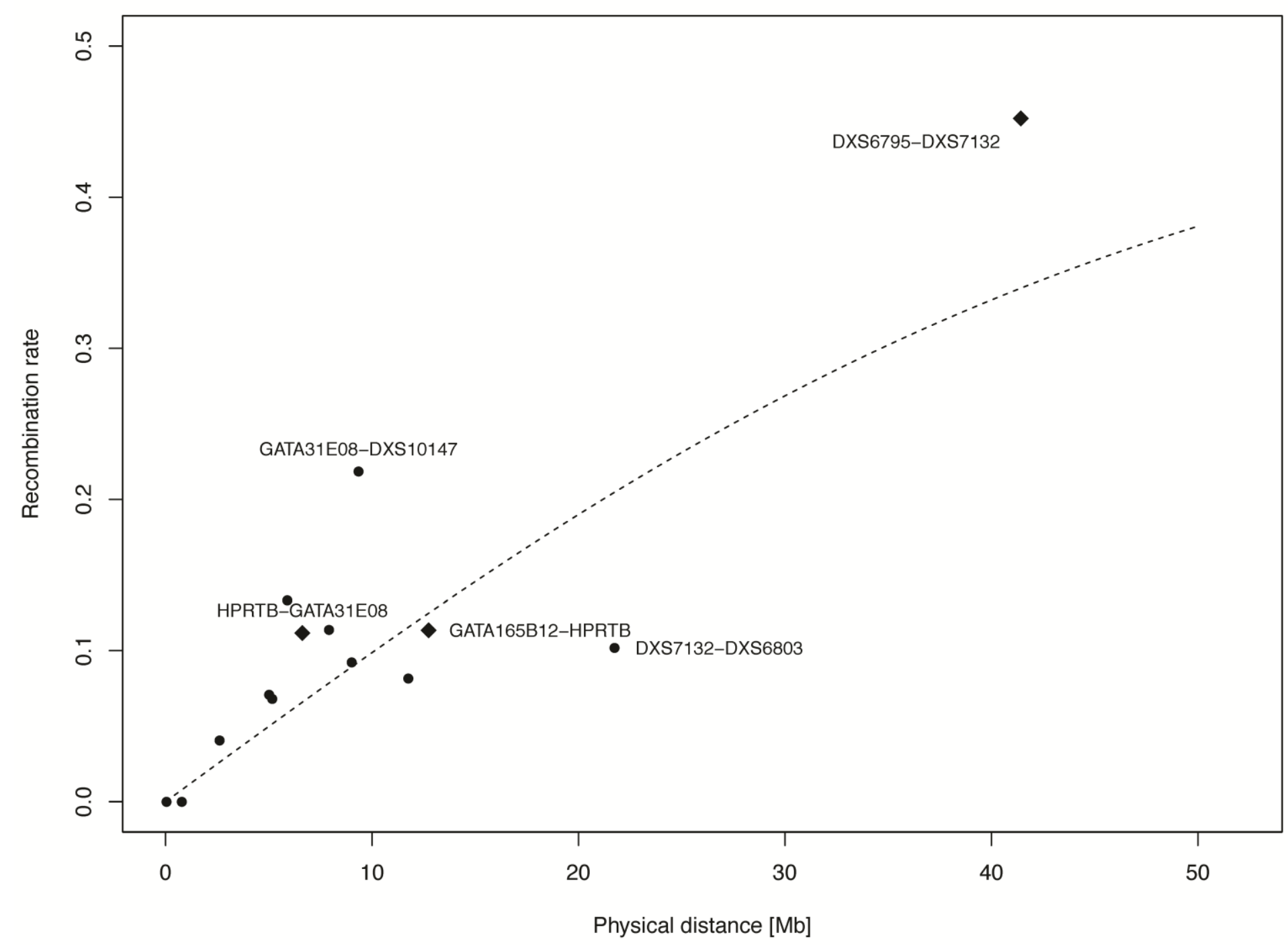




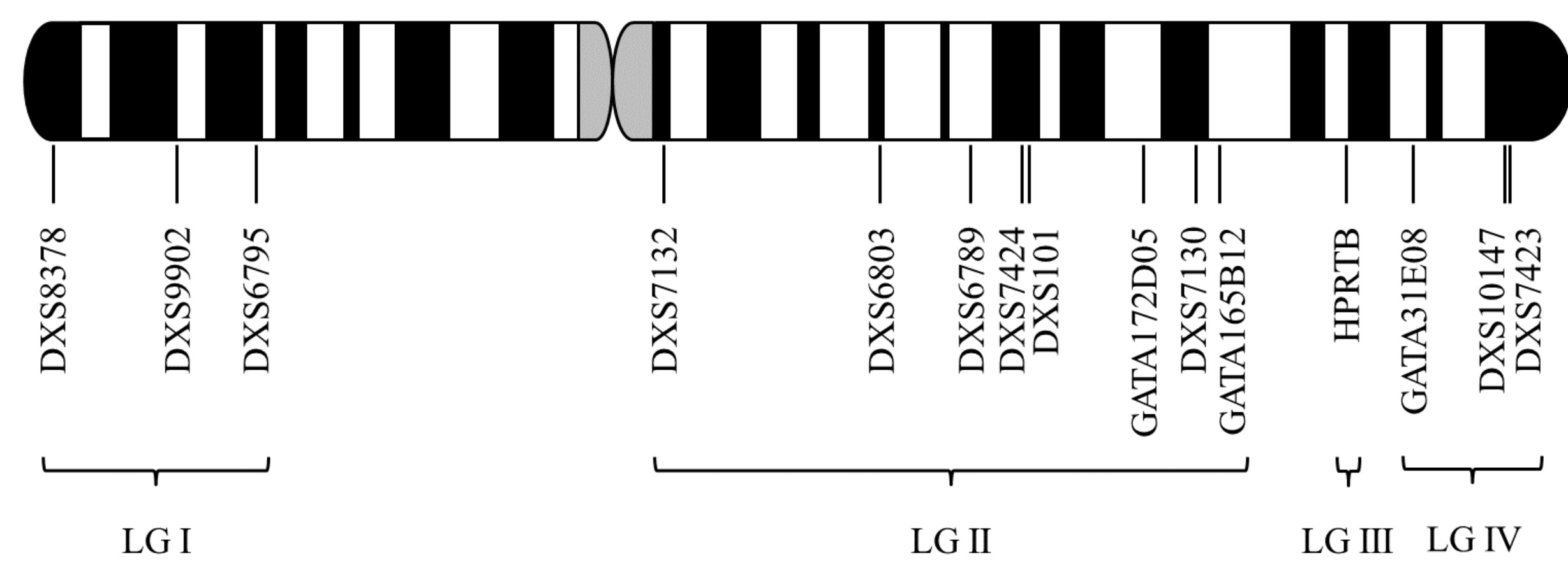

